\title{
ACCURACY OF USING DIFFERENT VOXEL SIZES TO DETECT OSSEOUS DEFECTS IN MANDIBULAR CONDYLE
}

\author{
Mehmet H. Kurt' ${ }^{1}$ Poyzan Bozkurt² , Cansu Görürgöz' , Batuhan Bakırarar ${ }^{3}$, Kaan Orhan ${ }^{1,4}$ \\ 'Department of Dentomaxillofacial Radiology, Faculty of Dentistry, Ankara University, Turkey \\ ${ }^{2}$ Department of Oral and Maxillofacial Surgery, Faculty of Dentistry, Ankara University, Turkey \\ ${ }^{3}$ Department of Biostatistics, Faculty of Medicine, Ankara University, Turkey \\ ${ }^{4}$ Ankara University Medical Design Application and Research Center (MEDITAM), Ankara, Turkey
}

\begin{abstract}
INTRODUCTION: Clinical evaluation of temporomandibular disorders alone is insufficient and should be supported with radiological imaging modalities. Cone-beam computed tomography (CBCT) is the ideal method for evaluating bony components of temporomandibular joint.

Овјестіves: The aim of this study was to present the ability of different voxel sizes in identifying osseous defects of mandibular condyle using CBCT images.

MATERIAL AND METHODS: The study sample consisted of nine dry human skulls (12 temporomandibular joints), containing fabricated osseous defects of different sizes $(0,0.8,1$, and $1.6 \mathrm{~mm})$. The joints were imaged using five imaging protocols $\left(0.400,0.200,0.150,0.100\right.$, and $\left.0.075 \mathrm{~mm}^{3}\right)$. Evaluation of the fabricated defects was carried out by two different observers, who noted presence or absence of a defect. Kappa coefficients were calculated to assess the intraobserver and interobserver agreement for each setting. Sensitivity, specificity, accuracy, and positive and negative predictive values were used to compare the observers' performance according to the gold standard and different defect diameters. Statistical significance was set at $p<0.05$.

RESULTS: The best results for both observers in comparison with the gold standard were achieved at a voxel size of $0.075 \mathrm{~mm}^{3}$. The sensitivity, specificity, and accuracy generally increased with decreasing voxel size. Positive and negative predictive values increased with decreasing voxel size and increasing defect size.

Conclusions: Obtaining CBCT scans with voxel sizes of 0.100 or $0.075 \mathrm{~mm}^{3}$ should be considered for the evaluation of osseous defects of mandibular condyle. A voxel size of $0.100 \mathrm{~mm}^{3}$ may be preferable due to lower patient's irradiation dose.
\end{abstract}

KEY wORDS: temporomandibular joint, osseous defect, cone-beam computerized tomography, voxel size.

J Stoma 2020; 73, 5: 217-224

DOI: https://doi.org/10.5114/jos.2020.100646

\section{INTRODUCTION}

Temporomandibular disorder is a general term for disorders affecting the masticatory muscles, temporomandibular joint (TMJ), and related structures, such as the mandibular condyle, temporal bone, and articular disc $[1,2]$. Among these disorders, degenerative dis- eases are the most common pathologies affecting the TMJ [3]. Osseous abnormalities related to degenerative arthritis of the TMJ include cortical bone loss, flattening, erosion, osteophyte formation, and sclerosis. In addition, neoplastic and developmental disorders or other abnormalities caused by infections may occur in the TMJ [4].

\section{JOURNAL OF STOMATOLOGY CZASOPISMO STOMATOLOGICZNE}

AdDRESS FOR CORRESPONDENCE: Dr. Poyzan Bozkurt, Department of Oral and Maxillofacial Surgery, Ankara University, Faculty of Dentistry, Emniyet Mahallesi, Incitas Sokak, Yenimahalle, 06540, Ankara, Turkey, e-mail: poyzanbozkurt@hotmail.com

ReCEIVED: 27.06.2020 • ACCEPTED: 20.08.2020 • PUBLISHED: 30.10.2020 
Clinical examination alone was reported to be insufficient for the diagnosis of these disorders [5]. TMJ visualization aims to determine the degree of disease, follow the progress of degenerative changes, and interpret treatment response [6]. Diagnostic activities of different scanning techniques may vary. At present, panoramic radiography, conventional tomography, computed tomography (CT), and cone-beam computed tomography (CBCT) are used to assess the bony components, whereas the soft tissue components (discs) of the TMJ are evaluated using magnetic resonance imaging (MRI) [7].

CBCT is being increasingly used in TMJ studies because the superposition of structures with conventional radiography constitutes an important problem with regard to evaluation. CBCT allows for the evaluation of bony components, joint spaces, and pathologies in all dimensions without superposition and structural distortion. In addition, CBCT also provides higher spatial resolution and substantially lower radiation dose than multislice CT [7].

The ability of CBCT to display image quality is influenced by the inspected object, scanning unit, field of view (FOV), scanning time, tube voltage, milliamp (mA) setting, and voxel size, which indicates spatial resolution. Voxel size, consisting of depth, height, and breadth dimensions, is isotropic (i.e., the three parameters are equal), and is an important factor in the visualization times of CBCT units and the diagnostic quality of CBCT images. The importance of voxel size for CBCT examinations has been well-documented for various diseases [8]. While using different CBCT units, differences in image quality have been observed when the voxel size is changed [9]. High diagnostic value can be achieved with smaller voxel sizes for detecting small changes, such as root fractures and root resorptions [10-12]. The voxel size is one of the sources of non-threshold heterogeneity while detecting small changes, and there is no existing relationship between voxel size and diagnostic accuracy $[13,14]$. On the other hand, image noise decreases with increasing voxel size and thick-slice images demonstrate less noise than thinner slices. Moreover, voxel size has a direct impact on both image resolution and noise [15-17]. As observed, there are conflicting opinions about the effectiveness of voxel size. Therefore, it is important for clinicians to understand the importance of using appropriate parameters for a specific diagnostic task. For this reason, in the present study, all parameters, which may affect image quality were kept constant, and the effect of voxel size on the diagnosis of small osseous changes in the mandibular condyle was investigated.

\section{OBJECTIVES}

The aim of this study was to present the ability of different voxel sizes in identifying osseous defects of mandibular condyle using CBCT images.

\section{MATERIAL AND METHODS}

This article does not refer to any studies with human or animal subjects performed by any of the authors. The study was approved by the faculty institutional review board (decision number, 14/4).

\section{SAMPLE PREPARATION}

In the present study, 12 non-damaged TMJ condyles from nine human dry skulls obtained from the faculty's inventory were used. Inclusion criteria for the skulls were defined as the presence of intact joint condyles in each skull, no evidence of any major bone pathology of the condyle and fossa, and sufficient dentition to preserve and stabilize the intercuspal position of the jaws. The exclusion criteria were damaged joints, major bone pathology, and unstable intercuspal position of the jaws.

Each joint surface was divided into eight regions: anteromedial, antero-mid-medial, antero-mid-lateral, anterolateral, posteromedial, postero-mid-medial, postero-mid-lateral, and posterolateral (Figure 1A). Due to narrower anatomy of the posteromedial condylar area, one of the condyles was divided into seven regions: anteromedial, antero-mid-medial, antero-mid-lateral, anterolateral, postero-mid-medial, postero-mid-lateral, and postero-lateral. A total of 95 regions were examined to evaluate the defects.

A dentomaxillofacial radiologist, who was not among the observers of the study, created osseous defects using a straight handpiece (Alegra; W\&H Dentalwerk, Bürmoos, Austria) and round carbide drill burs (G\&Z Instrumente $\mathrm{GmbH}$, Lustenau, Austria) with different diameters $(0.8,1$, and $1.6 \mathrm{~mm})$. These simulated defects were created by inserting the drill bur until maximum diameter hole was achieved. The defect sizes in each region were selected randomly and were completed according to the planned numbers of diameters: $n=24$ for $0.8-\mathrm{mm}$ defects, $n=24$ for 1 -mm defects, $n=24$ for $1.6-\mathrm{mm}$ defects, and $n=23$ for regions with no defects, which were used as controls (Figure 1B). To prevent dental wax from entering into the created defects, all condyles were covered with glove pieces, and small 2-mm thick dental wax pieces were used to mimic the joint space (Figure 1C). Mandibles were adjusted to the skulls in the maximum intercuspal position, and to replicate soft tissue attenuation, the skulls were coated with 2-mm thick dental wax (Figure 1D) and placed in a water-filled plastic container (Figure 2).

\section{CONE-BEAM COMPUTED TOMOGRAPHY IMAGING}

The skulls were imaged using Planmeca ProMax 3D Max CBCT unit with $96 \mathrm{kVp}$ and $8 \mathrm{~mA}$ settings (ProMax 3D Max; Planmeca, Helsinki, Finland). All skulls were scanned with a 50 x $55 \mathrm{~mm}$ FOV. All skulls were posi- 

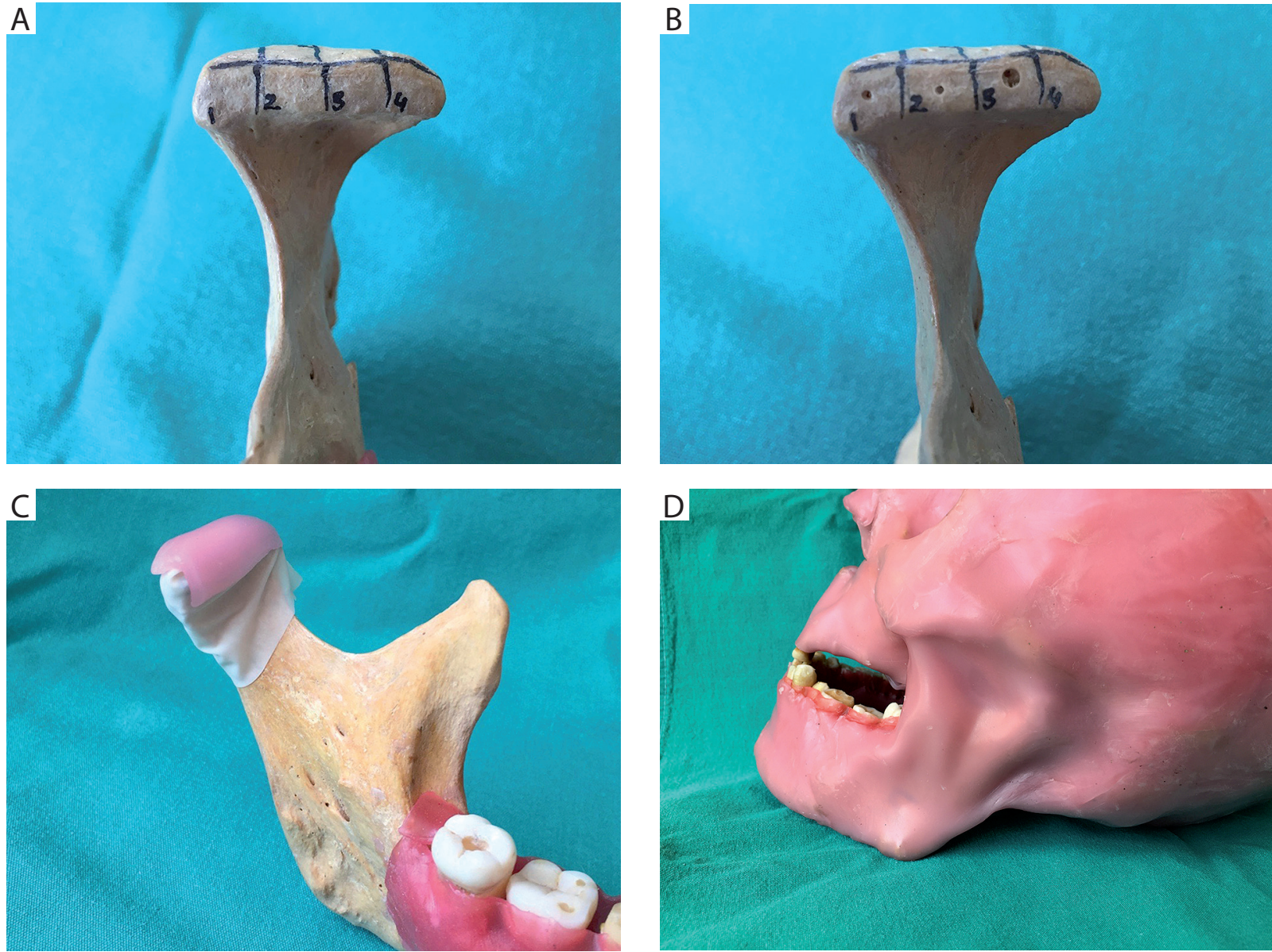

FIGURE 1. Condyle and skull preparation. A) Preparation of the condylar areas. B) Preparation of the defects. C) Preparation of the temporomandibular joint. D) Final vision of the skull after waxing
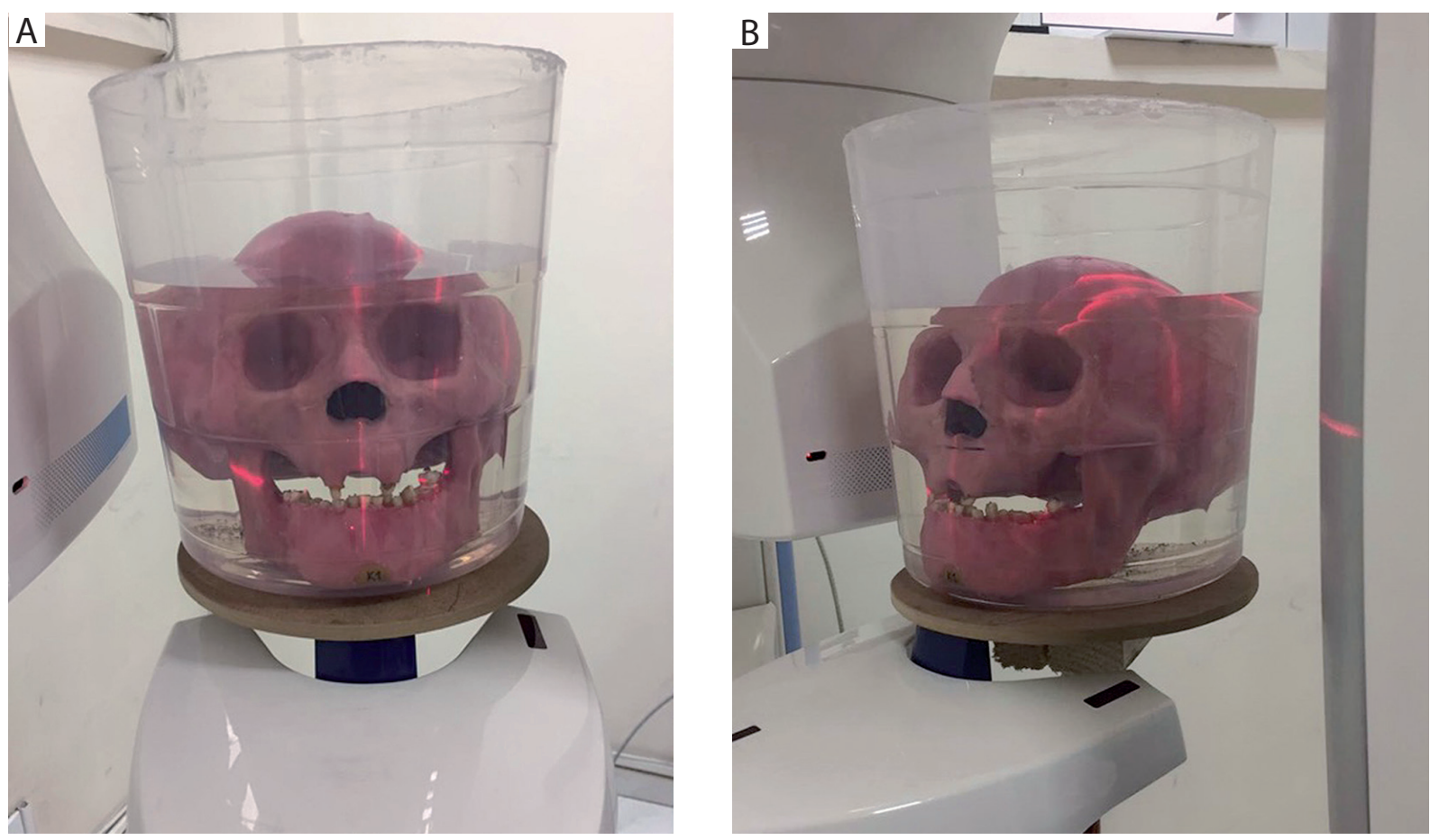

FIGURE 2. Replicating soft-tissue attenuation during imaging 



FIGURE 3. View of cone-beam computed tomography images. A) View of the 1.6-mm defect at a voxel size of $0.400 \mathrm{~mm}^{3}, 0.200 \mathrm{~mm}^{3}, 0.150 \mathrm{~mm}^{3}, 0.100 \mathrm{~mm}^{3}$, or $0.075 \mathrm{~mm}^{3}$. B) View of the 1-mm defect at a voxel size of $0.400 \mathrm{~mm}^{3}$, $0.200 \mathrm{~mm}^{3}, 0.150 \mathrm{~mm}^{3}, 0.100 \mathrm{~mm}^{3}$, or $0.075 \mathrm{~mm}^{3}$. C) View of the $0.8-\mathrm{mm}$ defect at a voxel size of $0.400 \mathrm{~mm}^{3}$, $0.200 \mathrm{~mm}^{3}, 0.150 \mathrm{~mm}^{3}, 0.100 \mathrm{~mm}^{3}$, or $0.075 \mathrm{~mm}^{3}$. D) View of the absent defect at a voxel size of $0.400 \mathrm{~mm}^{3}$, $0.200 \mathrm{~mm}^{3}, 0.150 \mathrm{~mm}^{3}, 0.100 \mathrm{~mm}^{3}$, or $0.075 \mathrm{~mm}^{3}$

tioned individually, with the TMJ located in the center of imaging field. In this FOV, TMJ scanning was performed with five different voxel sizes $(0.400,0.200,0.150$, 0.100 , and $0.075 \mathrm{~mm}^{3}$ ) provided by the CBCT device (Figure 3).

\section{EVALUATION OF CONE-BEAM COMPUTED \\ TOMOGRAPHY IMAGES}

Evaluation of the images was performed using sagittal and coronal slices along the longitudinal axis of mandibular condyle. Both observers were not limited during the evaluation of images including slice thickness and inter-slice intervals. They performed the evaluations with no time constraint in a dimly lit room.

Romexis 3.7 program (Planmeca) running on an NEC MultiSync MD215MG medical display (NEC, Munich, Germany) was used for evaluations. The medical display was on a 21.3-inch flat panel, color active-matrix TFT display, with a resolution of $2048 \times 2560$ at $75 \mathrm{~Hz}$ and a $0.17-\mathrm{mm}$ dot pitch, operated at 11.9 bits. This software enabled the reconstruction and observation of images in the sagittal, axial, and coronal planes.

A dentomaxillofacial radiologist (observer-1) and a maxillofacial surgeon (observer-2) independently analyzed all CBCT images for the presence or absence of defects. The observers were blinded to the locations and 
sizes of the simulated defects. In a dimly lit room, the examiners could change the image brightness, contrast, and magnification to their preferred ideal visual conditions for accurate diagnosis. Each observer reassessed the same images after an interval of 2 weeks to investigate interobserver and intraobserver reliability.

\section{STATISTICAL ANALYSIS}

Data analysis was performed using SPSS 11.5 for Windows (IBM Corporation, Armonk, NY, USA). Kappa coefficients were calculated to assess the intraobserver and interobserver agreement for each setting. Kappa values were interpreted according to the following criteria: 0.10 - no agreement, 0.11-0.40 - poor agreement, 0.41-0.60 moderate agreement, 0.61-0.80 - strong agreement, and 0.81-1.00 - excellent agreement [18]. Sensitivity, specificity, accuracy, and positive and negative predictive values were used to compare the evaluators' performance according to the gold standard and different defect diameters. In all analyses, $p<0.05$ was considered as statistically significant.

\section{RESULTS}

Intraobserver agreements are presented in Table 1. Strong agreement was achieved for both observers with voxel sizes of 0.100 and $0.075 \mathrm{~mm}^{3}$ ( 0.682 and 0.688 , and 0.761 and 0.695 , respectively), and the highest agreement was achieved with a voxel size of $0.075 \mathrm{~mm}^{3}$ for the first observer.
Interobserver agreement was calculated based on both readings (Table 2 ). Although the $\kappa$ value was higher at a voxel size of $0.100 \mathrm{~mm}^{3}$ for first reading ( 0.651 ; strong agreement), the second reading $\kappa$ value was higher at a voxel size of $0.075 \mathrm{~mm}^{3}$ (0.693; strong agreement). All interobservers' $\kappa$ values increased for the second reading.

The compliance of the two readings for both observers with the gold standard at different voxel sizes and evaluation criteria are presented in Table 3. The best results for both observers were achieved at a voxel size of $0.075 \mathrm{~mm}^{3}$. In addition, good agreement was observed at a voxel size of $0.075 \mathrm{~mm}^{3}$ for the first observer. Generally, all $\kappa$ values increased with decreasing voxel size.

Sensitivity, specificity, accuracy, and positive and negative predictive values of the different voxel sizes for different defect sizes are presented in Table 4 . The results showed that the sensitivity, specificity, accuracy, positive and negative predictive values increased as the defect size increased at all voxel sizes for both observers. As the voxel size increased, the sensitivity, specificity, accuracy, and positive and negative predictive values also increased for all defect sizes for both the observers.

\section{DISCUSSION}

Radiation dose is one of the most important factors to consider when applying imaging methods with ionizing radiation, but the obtained image quality and diagnostic sufficiency are also crucial. These two determinants influence both the correct diagnosis and treatment planning.

TABLE 1. Intraobserver agreements

\begin{tabular}{|c|c|c|c|c|c|c|}
\hline \multirow[t]{2}{*}{ Voxel size } & \multicolumn{3}{|c|}{ First observer } & \multicolumn{3}{|c|}{ Second observer } \\
\hline & $\kappa$ coefficient & S.E. & $p$-value & $\kappa$ coefficient & S.E. & $p$-value \\
\hline $0.400 \mathrm{~mm}^{3}$ & 0.222 & 0.097 & 0.015 & 0.181 & 0.102 & 0.041 \\
\hline $0.200 \mathrm{~mm}^{3}$ & 0.567 & 0.088 & $<0.001$ & 0.522 & 0.096 & $<0.001$ \\
\hline $0.150 \mathrm{~mm}^{3}$ & 0.665 & 0.085 & $<0.001$ & 0.546 & 0.138 & $<0.001$ \\
\hline $0.100 \mathrm{~mm}^{3}$ & 0.682 & 0.082 & $<0.001$ & 0.688 & 0.098 & $<0.001$ \\
\hline $0.075 \mathrm{~mm}^{3}$ & 0.761 & 0.071 & $<0.001$ & 0.695 & 0.095 & $<0.001$ \\
\hline
\end{tabular}

TABLE 2. Interobserver agreements

\begin{tabular}{|c|c|c|c|c|c|c|}
\hline \multirow[t]{2}{*}{ Voxel size } & \multicolumn{3}{|c|}{ First reading } & \multicolumn{2}{|c|}{ Second reading } & \\
\hline & к coefficient & S.E. & $p$-value & к coefficient & S.E. & $p$-value \\
\hline $0.400 \mathrm{~mm}^{3}$ & 0.204 & 0.107 & 0.036 & 0.165 & 0.088 & 0.040 \\
\hline $0.200 \mathrm{~mm}^{3}$ & 0.331 & 0.109 & 0.001 & 0.486 & 0.078 & $<0.001$ \\
\hline $0.150 \mathrm{~mm}^{3}$ & 0.376 & 0.100 & $<0.001$ & 0.512 & 0.105 & $<0.001$ \\
\hline $0.100 \mathrm{~mm}^{3}$ & 0.526 & 0.084 & $<0.001$ & 0.687 & 0.094 & $<0.001$ \\
\hline $0.075 \mathrm{~mm}^{3}$ & 0.651 & 0.092 & $<0.001$ & 0.693 & 0.081 & $<0.001$ \\
\hline
\end{tabular}


TABLE 3. Compliance levels of two observations with the gold standard at different voxel values and evaluation criteria

\begin{tabular}{|c|c|c|c|c|c|c|c|}
\hline Voxel size & Sensitivity & Specificity & Accuracy & PPV & NPV & к coefficient & $p$-value \\
\hline \multicolumn{8}{|l|}{ First observer } \\
\hline $0.400 \mathrm{~mm}^{3}$ & 79.2 & 43.5 & 70.5 & 81.4 & 40.0 & 0.220 & 0.032 \\
\hline $0.200 \mathrm{~mm}^{3}$ & 79.2 & 50.0 & 75.8 & 87.7 & 65.2 & 0.402 & $<0.001$ \\
\hline $0.150 \mathrm{~mm}^{3}$ & 88.9 & 65.2 & 83.2 & 88.9 & 65.2 & 0.541 & $<0.001$ \\
\hline $0.100 \mathrm{~mm}^{3}$ & 84.7 & 95.7 & 87.4 & 98.4 & 66.7 & 0.700 & $<0.001$ \\
\hline $0.075 \mathrm{~mm}^{3}$ & 88.9 & 100.0 & 91.6 & 100.0 & 74.2 & 0.795 & $<0.001$ \\
\hline \multicolumn{8}{|l|}{ Second observer } \\
\hline $0.400 \mathrm{~mm}^{3}$ & 88.9 & 43.5 & 77.9 & 83.1 & 55.6 & 0.350 & 0.001 \\
\hline $0.200 \mathrm{~mm}^{3}$ & 86.1 & 52.2 & 83.2 & 86.4 & 63.0 & 0.567 & $<0.001$ \\
\hline $0.150 \mathrm{~mm}^{3}$ & 95.8 & 60.9 & 86.3 & 88.8 & 85.7 & 0.570 & $<0.001$ \\
\hline $0.100 \mathrm{~mm}^{3}$ & 97.2 & 73.9 & 89.5 & 91.2 & 85.7 & 0.675 & $<0.001$ \\
\hline $0.075 \mathrm{~mm}^{3}$ & 98.6 & 78.3 & 91.6 & 93.2 & 93.3 & 0.764 & $<0.001$ \\
\hline
\end{tabular}

The relation between the radiation dose and acquired image quality in determining the appropriate imaging protocol, which leads to correct diagnosis must be properly established. The ALADA principle ("as low as diagnostically acceptable") lies at the heart of this situation, and decisions must be made wisely.

There are benefits from limiting the irradiation of FOV area at the anatomical region of interest, as scattered radiation decreases with reduced FOV resulting in less noise, fewer artifacts in images, and improved image quality. Also, a reduction in FOV is usually associated with a reduction of the patient's radiation dose [19], and reconstructions obtained with a larger FOV are less sharp due to greater beam angulation $[14,20]$. In the present study, the smallest FOV, in which the TMJ elements could be fully examined was used to achieve adequate assessment of artificial defects. While the FOV was kept constant, the effects of different voxel sizes on CBCT images were investigated.

To evaluate osseous defects of mandibular condyle, artificial defects were created with round burs. Utumi et al. [21] and Marques et al. [22] also simulated osseous defects on dry human skulls with three round carbide drill burs of different diameters, and this method was considered ideal to compare the observer's results and for statistical evaluation. Moreover, Librizzi et al. [4] and Patel et al. [23] created osseous defects with round burs to simulate osseous defects. Considering these results of previous studies, round burs were preferred to create artificial defects in the present study.

To the best of our knowledge, there have been few studies investigating the effects of voxel size for detecting osseous defects in the TMJ condyles [4, 14, 21, 25]. Patel et al. [21] investigated the accuracy of CBCT images in diagnosing condylar erosion defects of different sizes obtained with voxel sizes of 0.200 and $0.400 \mathrm{~mm}^{3}$. They reported that detecting extremely small defect sizes of $<2$-mm with a voxel size of $0.400 \mathrm{~mm}^{3}$ was more difficult, and that smaller FOV and voxel sizes provided more accurate information regarding the detection of TMJ erosions. Librizzi et al. [4] evaluated the detection accuracy of CBCT images reconstructed with different FOV and voxel sizes for condylar erosions. They concluded that a 6-inch FOV with a voxel size of $0.200 \mathrm{~mm}^{3}$ was significantly better than a 12 -inch FOV with a voxel size of $0.400 \mathrm{~mm}^{3}$. In contrast to these studies, Zhang et al. [25] reported no significant differences between normal and high-resolution images (voxel sizes of 0.160 , $0.200,0.300$, and $0.320 \mathrm{~mm}^{3}$ ) obtained using two different CBCT units. In addition, they concluded that the accuracy of detecting the condylar defects varied between CBCT units. Similarly, Lukat et al. reported that when images were obtained at native and down-sampled voxel resolutions, the results indicated that the detection of these osseous changes was not influenced by voxel size. The authors also reported that the detection of smaller defects was easier at higher spatial resolutions and suggested that a detection of larger defects would be less dependent on voxel size [14].

In the present study, there was a cumulative increase in the success rates with decreasing voxel size from $0.400 \mathrm{~mm}^{3}$ to $0.075 \mathrm{~mm}^{3}$. The observers were least successful at a voxel size of $0.400 \mathrm{~mm}^{3}$, whereas their most successful evaluations were at a voxel size of $0.075 \mathrm{~mm}^{3}$. Intraobserver (observer-1, 0.222-0.761; observer-2, 0.181-0.695) and interobserver agreements (first reading, 0.204-0.651; second reading, 0.165-0.693) increased from a poor rating to a good rating when voxel size decreased. Although the image quality is negatively affected due to increasing noise when the voxel size decreases [15-17], the current study obtained better diagnostic results at lower voxel sizes. However, the evaluations turn out to be much more challenging, and the observers evaluated lower voxel images with higher concentration 
levels during much longer periods. High level of diagnostic accuracy in lower voxel sizes regardless of noise can be attributed to this situation.

Marques et al. [23], Tsiklasis et al. [26], and Hussain et al. [27] observed that evaluation was becoming more challenging when decreasing the size of defect. Consistent with these studies, the most successful rates were achieved for $1.6-\mathrm{mm}$ defects, followed by $1.0-\mathrm{mm}$ defects, but the success rate was low for $0.8-\mathrm{mm}$ defects for both the observers in the present study. Therefore, larger defects could be less dependent on voxel size. These results were consistent with the literature and indicated that the selection of voxel size can be useful in the detection of small osseous defects of the condyle. Smaller voxel sizes improve spatial resolution, i.e., increases in spatial resolution can improve the ability to examine very small distances between objects. The size of a defect can affect diagnostic performance regardless of the voxel size.

\section{STUDY LIMITATIONS}

The artificial defects created with round burs had sharp edges and could not fully imitate real osseous defects. However, the results of this study can guide the clinicians in evaluating osseous changes in the mandibular condyle. Another limitation of this study was that only condylar defects could be evaluated, and osseous changes, such as flattening, sclerosis, decortication, osteophytes, and subchondral cysts could not be investigated. On the other hand, smaller osseous defects of the mandibular condyle can be correlated with the first stages of degenerative changes. Thus, the detection of these smaller osseous defects at an early stage can affect the prognosis of TMJ disease. In addition, in the setup of the present study, the major factor that caused deterioration of image resolution, i.e., patient motion, could not be taken into consideration. The radiation dose and exposure time increase as the resolution increases. Therefore, results from living patients may differ from those obtained in the present study. Marques et al. [23] used two different CBCT imaging protocols and concluded that, although not statistically significant, a multi-planar reconstruction protocol led to better results. The results of the present study were obtained using one device with only one protocol. Further research with different CBCT units and protocols is necessary.

In conclusion, the results of the present study showed that voxel sizes of 0.100 or $0.075 \mathrm{~mm}^{3}$ led to high success rates, and the most successful results were obtained at a voxel size of $0.075 \mathrm{~mm}^{3}$. Although these results did not mimic real clinical conditions, it may be advisable to use voxel size of $0.100 \mathrm{~mm}^{3}$ instead of $0.075 \mathrm{~mm}^{3}$ to examine osseous defects in the condyle, since a decreased voxel size requires a longer irradiation time.

\begin{tabular}{|c|c|c|c|c|c|c|c|c|c|c|c|c|c|}
\hline & å & & fi & ڤ્m & oे & $\bar{\delta}$ & ma & & $\stackrel{a}{\grave{m}}$ & $\stackrel{\sim}{\sim}$ & $\mid \begin{array}{l}\infty \\
\dot{m}\end{array}$ & $\stackrel{\sim}{\frac{1}{m}}$ & $\stackrel{\infty}{m}$ \\
\hline & $\widetilde{\Sigma}$ & & $\dot{s}$ & $\begin{array}{l}\infty \\
\stackrel{m}{m}\end{array}$ & $\frac{\mathscr{g}}{m}$ & 商 & $\overline{\widetilde{m}}$ & & $\stackrel{n}{\lesssim}$ & $\stackrel{+}{\infty}$ & $\begin{array}{l}\infty \\
\infty \\
\infty\end{array}$ & ò & $\bar{m}$ \\
\hline & $\overline{0}$ & & : & 오. & $\underset{\mathbb{N}}{\widetilde{N}}$ & $\stackrel{\circ}{\sim}$ & ๖े & & $\ddot{\nabla}$ & $\stackrel{n}{\approx}$ & $\stackrel{\sim}{\check{\sim}}$ & $\begin{array}{l}\infty \\
\stackrel{\infty}{\sim}\end{array}$ & 穴 \\
\hline & 8 & & t. & $\widehat{\infty}$ & $\begin{array}{l}\stackrel{a}{ } \\
\infty \\
\infty\end{array}$ & $\underset{\infty}{\circ}$ & ठ் & & $\bar{\infty}$ & $\stackrel{\sim}{\pi}$ & t্் & $\begin{array}{l}\infty \\
\infty \\
\infty\end{array}$ & ๙ָ. \\
\hline & ma & & S. & $\hat{\varnothing}$ & $\stackrel{\circ}{\circ}$ & 용 & ㅇ. & & $\stackrel{\circ}{8}$ & ㅇ. & 임 & $\stackrel{\circ}{\circ}$ & 웅 \\
\hline & $N$ & & Pُ & ஜ் & 용 & 용 & 요 & & $\begin{array}{l}9 \\
\infty \\
\infty\end{array}$ & مٌ & $\stackrel{\circ}{8}$ & 용 & 응 \\
\hline & $\overline{0}$ & & : & $\tilde{\vartheta}$ & ำ & $\overline{8}$ & $\stackrel{\sim}{\mathbb{N}}$ & & $\widehat{8}$ & ஓं & 岕 & $\tilde{m}$ & ڤ્ळ \\
\hline & 8 & & نج & $\stackrel{\circ}{\circ}$ & 오 & $\widehat{\delta}$ & $\underset{\mathbb{N}}{\stackrel{N}{N}}$ & & $\begin{array}{l}0 \\
\stackrel{i}{i} \\
\text { Ln }\end{array}$ & $\stackrel{\text { ஜூ }}{ }$ & ஸे & ñ & బิ \\
\hline & g & & ?ุ & డ్ర & 笍 & oి & Бே & & $\stackrel{m}{q}$ & 怠 & $\overline{\dot{\sigma}}$ & $\overline{\mathscr{\gamma}}$ & 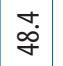 \\
\hline 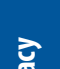 & $\mathbf{\Sigma}$ & & o. & $\overline{\mathscr{\gamma}}$ & 苗 & 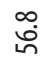 & $\begin{array}{c}9 \\
\infty \\
\infty\end{array}$ & & $\stackrel{\circ}{\stackrel{\rho}{m}}$ & $\stackrel{+}{\stackrel{b}{q}}$ & $\begin{array}{c}\stackrel{\sigma}{\infty} \\
\stackrel{m}{n}\end{array}$ & $\stackrel{\circ}{\dot{q}}$ & mo \\
\hline$\underline{z}$ & $\bar{\sigma}$ & & ְُ & $\stackrel{\nabla}{\sim}$ & $\hat{m}$ & 㐫 & F & & $\frac{0}{m}$ & 吕 & $\begin{array}{l}\infty \\
\stackrel{m}{n} \\
\stackrel{n}{n}\end{array}$ & oे & $\check{\dot{\sigma}}$ \\
\hline & 0 & & $?$ & $\stackrel{\infty}{\stackrel{\infty}{N}}$ & $\underset{\infty}{\tilde{\infty}}$ & $\underset{\infty}{\stackrel{\Delta}{\infty}}$ & $\begin{array}{l}0 \\
\dot{a}\end{array}$ & & $\stackrel{\circ}{\Sigma}$ & $\stackrel{\text { ๓ }}{\infty}$ & m̊. & นุ & б̆ \\
\hline & $m$ & & గુ & 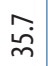 & $\stackrel{+}{\stackrel{\dot{\sigma}}{*}}$ & $\bar{夭}$ & $\underset{f}{\mathscr{f}}$ & & 气⿱丶万⿱⿰㇒一乂 & $\begin{array}{l}\dot{0} \\
\stackrel{\infty}{m}\end{array}$ & ㅇ. & $\stackrel{\nabla}{\stackrel{亠}{\sim}}$ & ㅇ. \\
\hline$\Rightarrow$ & $\mathbf{\Xi}$ & & $\frac{?}{n}$ & ָุ' & $\stackrel{\sim}{\stackrel{n}{m}}$ & $\bar{\Re}$ & $\begin{array}{l}\infty \\
\dot{f} \\
\dot{f}\end{array}$ & & $\widetilde{\Xi}$ & 户্户 & ナ̆ & 足 & ָั \\
\hline 흔 & 5 & & $\dot{\bar{v}}$ & $\hat{\sigma}$ & $\begin{array}{l}\infty \\
\stackrel{\sim}{\sim}\end{array}$ & $\frac{0}{m}$ & $\underset{\sim}{\stackrel{\sim}{\sim}}$ & & oి & 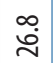 & ơ & $\hat{\sigma}$ & 讨 \\
\hline & 8 & & $\sigma^{2}$ & ָุ' & జี่ & นู่ & ○் & & $\stackrel{\stackrel{n}{F}}{\forall}$ & $\stackrel{\leftrightarrow}{\sim}$ & ָี & 亏ें & $\underset{\infty}{\infty}$ \\
\hline & $m$ & & b. & ஜ̊ं & ○் & ¿̊. & ठ் & & @̊ & @̊ & $\stackrel{0}{0}$ & $\stackrel{\circ}{0}$ & $\stackrel{\circ}{0}$ \\
\hline 穾 & & & نُ & $\stackrel{\circ}{\infty}$ & ஜ் & ¿ & ठ். & & na. & $\frac{m}{\sigma}$ & $\stackrel{\circ}{\circ}$ & ○் & ○் \\
\hline जั & - & & $\begin{array}{l}\text { ý } \\
\text { fin }\end{array}$ & $\underset{\sim}{\stackrel{\sim}{n}}$ & 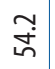 & ธิْ & $\widehat{\overbrace{}}$ & & 웃 & $\hat{8}$ & $\begin{array}{l}n \\
\infty \\
\infty\end{array}$ & $\hat{\check{\sigma}}$ & $\begin{array}{l}\infty \\
\text { ֵૂં }\end{array}$ \\
\hline & 요 & & पू' & నุ & $\begin{array}{l}\stackrel{a}{0} \\
\infty\end{array}$ & 广্ঠ & $\begin{array}{l}9 \\
\infty \\
\infty\end{array}$ & & $\begin{array}{l}\stackrel{a}{\infty} \\
\infty\end{array}$ & $\bar{\infty}$ & $\stackrel{N}{~}$ & $\begin{array}{l}6 \\
\infty \\
\infty\end{array}$ & $\begin{array}{l}\infty \\
\text { \&ू் }\end{array}$ \\
\hline$\frac{\frac{N}{4}}{\frac{\pi}{0}}$ & & & '. &  & 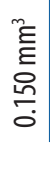 & 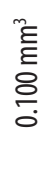 & $\mid \begin{array}{c}\tilde{E} \\
\underline{E} \\
\mathfrak{n} \\
\tilde{O} \\
0\end{array}$ & 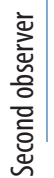 & 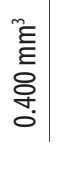 & 芯 & 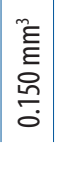 & 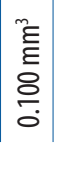 &  \\
\hline
\end{tabular}




\section{CONCLUSIONS}

Appropriate voxel sizes established to detect osseous defects of the temporomandibular joint will guide clinicians through the examination process of these diseases. According to the present study, voxel sizes of $0.100 \mathrm{~mm}^{3}$ and $0.075 \mathrm{~mm}^{3}$ appeared to have higher success rates in detecting osseous defects of the temporomandibular joint. A voxel size of $0.100 \mathrm{~mm}^{3}$ may be preferred because of lower irradiation dose. It can be mentioned that larger defects would be less dependent on voxel size.

\section{CONFLICT OF INTEREST}

The authors declare no potential conflicts of interest with respect to the research, authorship, and/or publication of this article.

\section{References}

1. Shaffer SM, Brismée JM, Sizer PS, Courtney CA. Temporomandibular disorders. Part 1: anatomy and examination/diagnosis. J Man Manip Ther 2014; 22: 2-12.

2. Wadhwa S, Kapila S. TMJ disorders: future innovations in diagnostics and therapeutics. J Dent Educ 2008; 72: 930-947.

3. Salemi F, Shokri A, Maleki FH, et al. Effect of field of view on detection of condyle bone defects using cone beam computed tomography. J Craniofac Surg 2016; 27: 644-648.

4. Librizzi ZT, Tadinada AS, Valiyaparambil JV, Lurie AG, Mallya SM. Cone-beam computed tomography to detect erosions of the temporomandibular joint: effect of field of view and voxel size on diagnostic efficacy and effective dose. Am J Orthod and Dentofacial Orthop 2011; 140: e25-e30.

5. Paesani D, Westesson PL, Hatala MP, Tallents RH, Brooks SL. Accuracy of clinical diagnosis for TMJ internal derangement and arthrosis. Oral Surg Oral Med Oral Pathol 1992; 73: 360-363.

6. Yadav S, Palo L, Mahdian M, Upadhyay M, Tadinada A. Diagnostic accuracy of 2 cone-beam computed tomography protocols for detecting arthritic changes in temporomandibular joints. Am J Orthod Dentofacial Orthop 2015; 147: 339-344.

7. Barghan S, Tetradis S, Mallya SM. Application of cone beam computed tomography for assessment of the temporomandibular joints. Aust Dent J 2012; 57 Suppl 1: 109-118.

8. Conticello S, Saita V, Ferlito S, Paterno A. Computed tomography in the study of the eustachian tube. Eur Arch Otorhinolaryngol 1989; 246: 259-261.

9. Spin-Neto R, Gotfredsen E, Wenzel A. Impact of voxel size variation on CBCT-based diagnostic outcome in dentistry: a systematic review. J Digit Imaging 2013; 26: 813-820.

10. Wenzel A, Haiter-Neto F, Frydenberg M, Kirkevang LL. Variableresolution cone-beam computerized tomography with enhancement filtration compared with intraoral photostimulable phosphor radiography in detection of transverse root fractures in an in vitro model. Oral Surg Oral Med Oral Pathol Oral Radiol Endod 2009; 108: 939-945.

11. Melo SLS, Bortoluzzi EA, Abreu M, Correa LR, Correa M. Diagnostic ability of a cone-beam computed tomography scan to assess longitudinal root fractures in prosthetically treated teeth. J Endod 2010; 36: 1879-1882.

12. Kolsuz ME, Bagis N, Orhan K, Avsever H, Demiralp KÖ. Comparison of the influence of FOV sizes and different voxel resolutions for the assessment of periodontal defects. Dentomaxillofac Radiol 2015; 44: 20150070.
13. Ma R, Yin S, Li G. The detection accuracy of cone beam CT for osseous defects of the temporomandibular joint: a systematic review and meta-analysis. Sci Rep 2016; 6: 34714.

14. Lukat TD, Perschbacher SE, Pharoah MJ, Lam EW. The effects of voxel size on cone beam computed tomography images of the temporomandibular joints. Oral Surg Oral Med Oral Pathol Oral Radiol 2015; 119: 229-237.

15. Elkhateeb SM, Torgersen GR, Arnout EA. Image quality assessment of clinically-applied CBCT protocols using a QAT phantom. Dentomaxillofac Radiol 2016; 45: 20160075.

16. Bamba J, Araki K, Endo A, Okano T. Image quality assessment of three cone beam CT machines using the SEDENTEXCT CT phantom. Dentomaxillofac Radiol 2013; 42: 20120445

17. Maloul A, Fialkov J, Whyne C. The impact of voxel size-based inaccuracies on the mechanical behavior of thin bone structures. Ann Biomed Eng 2011; 39: 1092-1100.

18. Landis JR, Koch GG. The measurement of observer agreement for categorical data. Biometrics 1977; 33: 159-174.

19. Scarfe WC, Li Z, Aboelmaaty W, Scott SA, Farman AG. Maxillofacial cone beam computed tomography: essence, elements and steps to interpretation. Aust Dent J 2012; 57 Suppl 1: 46-60.

20. Patcas R, Muller L, Ullrich O, Peltomaki T. Accuracy of conebeam computed tomography at different resolutions assessed on the bony covering of the mandibular anterior teeth. Am J Orthod Dentofacial Orthop 2012; 141: 41-50.

21. Hatcher DC. Operational principles for cone-beam computed tomography. J Am Dent Assoc 2010; 141: 3S-6S.

22. Utumi ER, Perrella A, Albuquerque MAP, Adde CA, Rocha RG Cavalcanti MG. Evaluation of simulated bone lesion in the head of the mandible by using multislice computed tomography. J Appl Oral Sci 2009; 17: 521-526.

23. Marques AP, Perrella A, Arita ES, Fenyo Soeiro de Matos Pereira M de Gusmão Paraíso Cavalcanti M. Assessment of simulated mandibular condyle bone lesions by cone beam computed tomography. Braz Oral Res 2010; 24: 467-474.

24. Patel A, Tee BC, Fields H, Jones E, Chaudhry J, Sun Z. Evaluation of cone-beam computed tomography in the diagnosis of simulated small osseous defects in the mandibular condyle. Am J Orthod Dentofacial Orthop 2014; 145: 143-156.

25. Zhang ZL, Shi XQ, Ma XC, Li G. Detection accuracy of condylar defects in cone beam CT images scanned with different resolutions and units. Dentomaxillofac Radiol 2014; 43: 20130414.

26. Tsiklakis K, Syriopoulos K, Stamatakis HC. Radiographic examination of the temporomandibular joint using cone beam computed tomography. Dentomaxillofac Radiol 2004; 33: 196-201.

27. Hussain AM, Packota G, Major PW, Flores-Mir C. Role of different imaging modalities in assessment of temporomandibular joint erosions and osteophytes: a systematic review. Dentomaxillofac Radiol 2008; 37: 63-71. 